\title{
Finding a Link between Randomness and Fuzziness
}

\author{
Fokrul Alom Mazarbhuiya \\ College of Computer Science and IT, Albaha University, Albaha, KSA \\ Email: fokrul_2005@yahoo.com
}

Received 5 March 2014; revised 5 April 2014; accepted 12 April 2014

Copyright (C 2014 by author and Scientific Research Publishing Inc.

This work is licensed under the Creative Commons Attribution International License (CC BY). http://creativecommons.org/licenses/by/4.0/

(c) (i) Open Access

\begin{abstract}
If sample realizations are intervals, if the upper and the lower boundaries of such intervals are realizations of two independently distributed random variables, the two probability laws together lead to some interesting assertions. In this article, we shall attempt to remove certain confusions regarding the relationship between probability theory and fuzzy mathematics.
\end{abstract}

\section{Keywords}

Probability, Possibility, Fuzzy Sets, Possibilistic Events, Uniform Probability Law, Triangular Fuzzy Number, Order Statistics

\section{Introduction}

Let $X$ and $Y$ be two random variables independently distributed real intervals $[a, b]$ and $[b, c]$ respectively. Let $F($.$) and G($.$) be the probability distribution functions of X$ and $Y$ respectively.

Let $\alpha \in[a, b]$ and $\beta \in[b, c]$ the juxtaposition of events $[\alpha, b]$ and $[b, \beta]$ would give us the interval $[\alpha, \beta]$. Observe that while $[\alpha, b]$ and $[b, \beta]$ are simple probabilistic events, $[\alpha, \beta]$ is totally reference as an event because its boundaries are from two probabilistic populations. For simplicity let us call $[\alpha, \beta]$ a twopopulation event (t.p.e.), we are interested to calculate the probability associated with such a t.p.e., and thereafter to explain its physical significance.

\section{Simple and Compound Two-Population Events}

Let $\mathrm{A}=[\alpha, \beta]$ be a t.p.e. It is easy to see that

$$
\operatorname{Prob}[\alpha \leq X \leq \beta]=(1-F(\alpha)) \cdot G(\beta)
$$


In other words, in terms of intervals

$$
\operatorname{Prob}[[b, b] \leq[X, Y] \leq[\alpha, \beta]]=(1-F(\alpha)) \cdot G(\beta)
$$

The identities (1) and (2) are rather elementary. But (2) in particular can have far reaching consequences, which we are going to assert later. The t.p.e. $[\alpha, \beta]$ is a simple t.p.e. in the sense, that if $A_{1}=\left[\alpha_{1}, \beta_{1}\right]$ and $A_{2}=\left[\alpha_{2}, \beta_{2}\right]$ are t.p.e.'s, we shall call $A_{1} \cup A_{2}$ a compound t.p.e.

Let $\alpha_{(1)}=\min \left(\alpha_{1}, \alpha_{2}\right) \quad \alpha_{(2)}=\max \left(\alpha_{1}, \alpha_{2}\right) \quad \beta_{(1)}=\min \left(\beta_{1}, \beta_{2}\right)$, and $\beta_{(2)}=\max \left(\beta_{1}, \beta_{2}\right)$.

It is easy to see that

$$
\operatorname{Prob}\left[\alpha_{1} \leq X \leq b \text {, or } \alpha_{2} \leq X \leq b\right]=\operatorname{Prob}\left[\alpha_{(1)} \leq X \leq b\right]
$$

Similarly

$$
\operatorname{Prob}\left[b \leq Y \leq \beta_{1} \text {, or } b \leq Y \leq \beta_{2}\right]=\operatorname{Prob}\left[b \leq X \leq \beta_{(1)}\right]
$$

Therefore for the compound t.p.e.

$$
\begin{aligned}
& \operatorname{Prob}\left[A_{1} \cup A_{2}\right] \\
& =\operatorname{Prob}\left[[b, b] \leq[X, Y] \leq\left[\alpha_{1}, \beta_{1}\right] \text { or }[b, b] \leq[X, Y] \leq\left[\alpha_{2}, \beta_{2}\right]\right] \\
& =\operatorname{Prob}\left[[b, b] \leq[X, Y] \leq\left[\alpha_{(1)}, \beta_{(1)}\right]\right] \\
& =\operatorname{Prob}\left[\alpha_{(1)} \leq X \leq b, \text { or } b \leq Y \leq \beta_{(1)}\right] \\
& =\left(1-F\left(\alpha_{1}\right)\right) \cdot G\left(\beta_{1}\right)
\end{aligned}
$$

In the same way,

$$
\operatorname{Prob}\left[\alpha_{1} \leq X \leq b \text {, and } \alpha_{2} \leq X \leq b\right]=\operatorname{Prob}\left[\alpha_{(2)} \leq X \leq b\right]
$$

Similarly

$$
\operatorname{Prob}\left[b \leq Y \leq \beta_{1} \text {, and } b \leq Y \leq \beta_{2}\right]=\operatorname{Prob}\left[b \leq X \leq \beta_{(1)}\right]
$$

Therefore,

$$
\begin{aligned}
& \operatorname{Prob}\left[A_{1} \cap A_{2}\right] \\
& =\operatorname{Prob}\left[[b, b] \leq[X, Y] \leq\left[\alpha_{1}, \beta_{1}\right] \text { and }[b, b] \leq[X, Y] \leq\left[\alpha_{2}, \beta_{2}\right]\right] \\
& =\operatorname{Prob}\left[[b, b] \leq[X, Y] \leq\left[\alpha_{(2)}, \beta_{(1)}\right]\right] \\
& =\operatorname{Prob}\left[\alpha_{(2)} \leq X \leq b, b \leq Y \leq \beta_{(1)}\right] \\
& =\left(1-F\left(\alpha_{2}\right)\right) \cdot G\left(\beta_{1}\right)
\end{aligned}
$$

Identities (3) and (4) give us the probabilities of union and intersection respectively of two simple two population events.

Our discussions on probability of a t.p.e. ends here. In what follows, we shall explain the physical significance of identity (2). It would thereafter be shown that a t.p.e. is indeed a possibilistic event.

\section{Physical Significance of a Two-Population Event}

Zadeh ([1]) introduced the mathematics of fuzziness long forty nine years ago. It has made inroads into almost all applied branches of knowledge. However, most of the mathematicians shy away from this branch of mathematics for various reasons. One such is the belief that randomness and fuzziness are two independent concepts.

In this section we shall show the following:

a) Randomness can actually be at the root of fuzziness.

b) Two independent probability laws can define a fuzzy number.

First, let us review certain necessary definitions. 


\subsection{Some Basic Definitions Related to Fuzziness}

Let $E$ be the universe of discourse. A fuzzy set $A$ in $E$ is characterized by a membership function $v_{A}(x)$ lying in $[0,1] . v_{A}(x)$ for $x \in E$ represents the grade of membership of $x$ in $A$. Thus a fuzzy set $A$ is defined as

$$
A=\left\{\left(x, v_{A}(x)\right), x \in E\right\}
$$

A fuzzy set $A$ is said to be normal if $v_{A}(x)=1$ for at least one $x \in E$

An $\alpha$-cut of a fuzzy set is an ordinary set of elements with membership grade greater than or equal to a threshold $\alpha, \quad 0 \leq \alpha \leq 1$. Thus an $\alpha$-cut $A_{\alpha}$ of a fuzzy set $A$ is characterized by [2]

$$
A_{\alpha}=\left\{x \in E, v_{A}(x) \geq \alpha\right\}
$$

A fuzzy set is said to be convex if all its $\alpha$-cuts are convex sets [2].

A fuzzy number is a convex normalized fuzzy set $A$ defined on the real line $E$ such that

i) there exists an $x_{0} \in E$ such that $v_{A}\left(x_{0}\right)=1$, and

ii) $v_{A}(x)$ is piecewise continuous.

Indeed, if $[a, c]$ is a real interval with $a<b<c$, for a fuzzy number denoted by $[a, b, c]$ with

$v_{A}(a)=v_{A}(c)=0$, and $v_{A}(b)=1 . v_{A}(x)$ for $x \in[a, b]$ is known as left reference function and $v_{A}(x)$ for $x \in[b, c]$ is known as the right reference function of the fuzzy number $\mathrm{A}=[a, b, c]$ (L-R fuzzy number) [3]. For such an L-R (left-right) fuzzy number, the left reference function is non-decreasing and the right reference function is non-increasing. Finally, the membership function of a fuzzy set is viewed as its possibility distribution [4].

\subsection{Set Superimposition}

When we overwrite, the overwritten portion looks darker. Indeed, the doubly represented portion looks doubly dark. To explain this, we would need a set operation (S) of superimposition of two sets $A$ and $B$ defined as:

$$
A(S) B=(A-B)(+)(A \cap B)^{(2)}(+)(B-A)
$$

where $(A \cap B)^{(2)}$ are the elements of $(A \cap B)$ represented twice, and $(+)$ represents union of disjoint sets.

If $A=\left[a_{1}, b_{1}\right]$ and $B=\left[a_{2}, b_{2}\right]$ are two real intervals such that $(A \cap B) \neq \phi$, we would get from (5)

$$
\left[a_{1}, b_{1}\right](S)\left[a_{2}, b_{2}\right]=\left[a_{(1)}, a_{(2)}\right](+)\left[a_{(2)}, b_{(1)}\right]^{(2)}(+)\left(b_{(1)}, b_{(2)}\right)
$$

where $a_{(1)}=\min \left(a_{1}, a_{2}\right), a_{(2)}=\max \left(a_{1}, a_{2}\right), b_{(1)}=\min \left(b_{1}, b_{2}\right)$, and $b_{(2)}=\max \left(b_{1}, b_{2}\right)$.

Identity (6) explains why-if two line segments are superimposed, the common portion looks doubly dark [5]. Mazarbhuiya et al. used this operation to define fuzzy arithmetic operations [6] and to solve a fuzzy equation [7]. Set superimposition is also used in mining temporal data ([8] [9]). In ([10] [11]), Baruah tries to establish link between probability law and possibility law.

Let now, $\left[a_{1}, b_{1}\right]^{(1 / 2)}$ and $\left[a_{2}, b_{2}\right]^{(1 / 2)}$ be two fuzzy sets with constant membership $1 / 2$ everywhere.

Following (6) we can write:

$$
\left[a_{1}, b_{1}\right]^{(1 / 2)}(S)\left[a_{2}, b_{2}\right]^{(1 / 2)}=\left[a_{(1)}, a_{(2)}\right]^{(1 / 2)}(+)\left[a_{(2)}, b_{(1)}\right]^{(1)}(+)\left[b_{(1)}, b_{(2)}\right]^{(1 / 2)}
$$

An obvious extension of (7) is: if $x_{(1)}, x_{(2)}, \cdots, x_{(n)}$ are values of $x_{1}, x_{2}, \cdots, x_{n}$ arranged in ascending order, and $y_{(1)}, y_{(2)}, \cdots, y_{(n)}$ are values of $y_{1}, y_{2}, \cdots, y_{n}$ are arranged in ascending order, we would get

$$
\begin{aligned}
& {\left[x_{1}, y_{1}\right]^{(1 / n)}(S)\left[x_{2}, y_{2}\right]^{(1 / n)}(S) \cdots(S)\left[x_{n}, y_{n}\right]^{(1 / n)} } \\
= & {\left[x_{(1)}, x_{(2)}\right]^{(1 / n)}(+)\left[x_{(2)}, x_{(3)}\right]^{(2 / n)}(+) \cdots(+)\left[x_{(r)}, x_{(r+1)}\right]^{(r / n)}(+) \cdots(+)\left[x_{(n)}, y_{(1)}\right]^{(1)} } \\
& (+) \cdots(+)\left[y_{(n-r)}, y_{(n-r+1)}\right]^{(r / n)}(+) \cdots(+)\left[y_{(n-2)}, y_{(n-1)}\right]^{(2 / n)}(+)\left[y_{(n-1)}, y_{(n)}\right]^{(1 / n)}
\end{aligned}
$$

At this point, we would need a classical result from order statistics to proceed further. 


\subsection{The Glivenko-Cantelli Theorem}

Define

$$
F_{n}(x)=\left\{\begin{array}{lc}
0, & x \prec x_{(1)} \\
\frac{r}{n}, & x_{(r)} \leq x \leq x_{(r+1)}, r=1,2, \cdots, n-1 \\
1, & x \succ x_{(n)}
\end{array}\right.
$$

where $x_{1}, x_{2}, \cdots, x_{n}$ are realizations of a random variable $X$, and $x_{(1)}, x_{(2)}, \cdots, x_{(n)}$ are $x_{1}, x_{2}, \cdots, x_{n}$ arranged in ascending order.

$F(x)$ is random here with

$$
\operatorname{Prob}\left[F_{n}(x)=\frac{r}{n}\right]=\frac{n !}{r !(n-r) !}(F(x))^{r}(1-F(x))^{n-r}
$$

where $F(x)$ is the underlying theoretical distribution function. $F_{n}(x)$ being an empirical distribution function.

In such a case, the Glivenko-Cantelli Theorem on order statistics [12] states that

$$
\operatorname{Prob}\left[\sup \left|F_{n}(x)-F(x)\right| \rightarrow 0\right]=1
$$

This means, $F_{n}(x) \rightarrow F(x)$ uniformly in $x$ with probability 1 .

\subsection{The Glivenko-Cantelli Theorem Applied to Superimposed Equally Fuzzy Sets}

Consider the identity (8) once again. Let $X$ and $Y$ be random and independently distributed in two real intervals $[a, b]$ and $[b, c]$ respectively. Let $F(*)$ and $G(*)$ be the probability distribution functions of $X$ and $Y$ respectively.

Application of Glivenko-Cantelli Theorem on identity (8) now after the imposition of randomness, as $n \rightarrow \infty$, on n superimposed equally fuzzy sets $\left[x_{i}, x_{(i+1)}\right], i=1,2, \cdots, n$, gives us the following:

i) $\operatorname{Prob}\left[\sup \left|\frac{r}{n}-F(x)\right| \rightarrow 0\right]=1, a \leq x \leq b$.

ii) $\operatorname{Prob}\left[\sup \left|\frac{r}{n}-(1-G(y))\right| \rightarrow 0\right]=1, b \leq y \leq c$

In other words, a probability distribution function $F(x)$ in $[a, b]$ can indeed be the left reference function, and a complementary probability distribution function or survival function $1-G(y)$ in $[b, c]$ can similarly be the right reference function of a fuzzy number.

We can therefore conclude that randomness can actually be at the root of fuzziness, and that two independent probability laws can define a fuzzy number.

\section{Discussions}

The possibility distribution function in $\Omega=[a, b, c]$, which indeed is the membership function of a fuzzy number $[a, b, c]$, can be viewed as two different functions, one in the interval $\Omega_{1}=[a, b]$ and the other in the interval $\Omega_{2}=[b, c]$. In $\Omega_{1}$, if a probability law $P \Omega_{1}$, and in $\Omega_{2}$, if another probability law $P \Omega_{2}$ are defined such that $\Omega_{1} \cup \Omega_{2}$ defines a fuzzy interval, $P \Omega_{1}$ and $P \Omega_{2}$ together can define the possibility distribution $F \Omega$ on $\Omega=\Omega_{1} \cup \Omega_{2}$. The fuzzy mathematicians have all along been trying to impose a $P \Omega$ on the same $\Omega$ on which $F \Omega$ is defined. For this mistake, they have all along been saying that fuzziness and randomness are two independent concepts.

In fact, every sample realization $b_{i}, i=1,2, \cdots, m$ can be thought of as values of unit fuzzy membership, around which intervals expressed as $\left[a_{1}, b_{1}, c_{1}\right]$ would give us fuzzy numbers. Accordingly, in $\left[a_{1}, b_{1}\right]$ there can be a probability law, while in $\left[b_{1}, c_{1}\right]$ there can be another probability law. Within the fuzzy number $\left[a_{1}, b_{1}, c_{1}\right]$ one can define possibilistic events, which are indeed t.p.e.'s defined in Section 1 of this article. Probabilities of such t.p.e.'s or possibilistic events, simple or compound, can be found following (2), (3) and (4). As long as one continues to impose a single probability law in a $\Omega$ where a possibility distribution is defined, the 
question of involving randomness to define fuzziness cannot arise. Independent probability laws on $\Omega_{1}$ and $\Omega_{2}$, can finally define a possibility law on $\Omega_{1} \cup \Omega_{2}$. In other words, possibility can indeed be a measure, and indeed it is a product measure.

It is to be noted that identities (2) and (10) are directly related. In fact, (10) explains the physical significance of (2). We would like to conclude our discussions with an important comment. The fuzzy mathematicians invariably use the triangular fuzzy number in computations. No fuzzy mathematician has as yet supplied a logical answer as to why they use the triangular fuzzy number only. An obvious answer hidden in escapism is that it is simple to use. Simplicity of the triangular number in fact lies elsewhere. If $F($.$) and G($.$) are uniform prob-$ ability distribution functions, we simply get the triangular number. What we mean is: just as the uniform law is the simplest in the field of probability, so is the triangular number the simplest in fuzzy mathematics.

In other words, the simplicity of the triangular fuzzy number is rooted at the simplicity of the uniform probability law. This is the actual reason why the triangular number is simple.

To illustrate the concept, let us take a simple example. Let $A=[a, b, c]$ be a normal fuzzy number whose membership function is given by

$$
A(x)= \begin{cases}F(x), & \text { for } a \leq x \leq b \\ G(x), & \text { for } b \leq x \leq c\end{cases}
$$

where

$$
F(x)= \begin{cases}0, & \text { for } x \prec a \\ \frac{x-a}{b-a}, & \text { for } a \leq x \leq b\end{cases}
$$

is the Dubois-Prade left reference function and

$$
G(x)= \begin{cases}0, & \text { for } x \succ c \\ \frac{x-b}{c-b}, & \text { for } b \leq x \leq c\end{cases}
$$

is the Dubois-Prade right reference function. If we observe clearly, then we find that $F(x)$ is actually a probability distribution function whose probability density function is $f(x)=\frac{1}{b-a}$ and $G(x)$ is a complementary probability distribution function or survival function whose probability density function is $g(x)=\frac{1}{c-b}$. This illustrates the fact that randomness can actually be at the root of fuzziness and two probability laws are sufficient to define a fuzzy number.

\section{Acknowledgements}

This work has been dedicated in honor of my beloved teacher Professor Hemanta K. Baruah, Former Dean of Science, Gauhati University, India and Current Vice Chancellor of Bodoland University, India.

\section{References}

[1] Zadeh, L.A. (1965) Fuzzy Sets as a Basis of Theory of Possibility. Fuzzy Sets and Systems, 1, 3-28. http://dx.doi.org/10.1016/0165-0114(78)90029-5

[2] Chen, G.Q., Lee, S.C. and Yu, E.S.H. (1983) Application of Fuzzy Set Theory to Economics, In: Wang, P.P., Ed., Advances in Fuzzy Sets, Possibility Theory, and Application, Plenum Press, New York, 277-305. http://dx.doi.org/10.1007/978-1-4613-3754-6 18

[3] Dubois, D. and Prade, H. (1983) Ranking Fuzzy Number in the Setting of Possibility Theory. Information Sciences, 3, 183-224. http://dx.doi.org/10.1016/0020-0255(83)90025-7

[4] Prade, H. (1983) Fuzzy Programming: Why and How? Some Hints and Examples, in Advances in Fuzzy Sets, Possibility Theory and Application.

[5] Baruah, H.K. (1999) Set Superimposition and Its Application to the Theory of Fuzzy Sets. Journal of the Assam Science Society, 40, 25-31.

[6] Mazarbhuiya, F.A., Mahanta, A.K. and Baruah, H.K. (2003) Fuzzy Arithmetic without Using the Method of $\alpha$-Cut. 
Bulletin of Pure and Applied Sciences, 22E, 45-54.

[7] Mazarbhuiya, F.A., Mahanta, A.K. and Baruah, H.K. (2011) Solution of the Fuzzy Equation A+X= B Using the Method of Superimposition. Applied Mathematics, 2, 1039-1045. http://dx.doi.org/10.4236/am.2011.28144

[8] Mahanta, A.K., Mazarbhuiya, F.A. and Baruah, H.K. (2008) Finding Calendar-Based Periodic Patterns. Pattern Recognition Letters, 29, Elsevier Publication, USA, 1274-1284.

[9] Mazarbhuiya, F.A. and Abulaish, M. (2012) Clustering Periodic Patterns Using Fuzzy Statistical Parameters. International Journal of Innovative Computing Information and Control (IJICIC), 8, 2113-2124.

[10] Baruah, H.K. (2010) The Randomness-Fuzziness Consistency Principle. International Journal of Energy, Information and Communications, 1, 37-48.

[11] Baruah, H.K. (2012) An Introduction to the Theory of Imprecise Sets: The Mathematics of Partial Presence. Journal of Mathematical and Computational Science, 2, 110-124.

[12] Loeve, M. (1977) Probability Theory I. Springer Verlag, New York. 